\title{
A comparative study of single-vs. double-row technique in surgical treatment of insertional Achilles tendinopathy
}

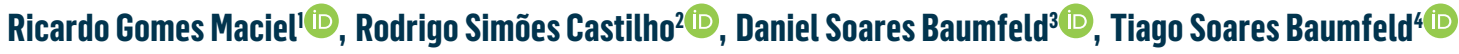 \\ 1. Hospital Marcio Cunha, Ipatinga, MG, Brazil. \\ 2. Hospital Mater Dei, Belo Horizonte, MG, Brazil. \\ 3. Hospital das Clínicas, universidade Federal de Minas Gerais, Belo Horizonte, MG, Brazil. \\ 4. Hospital Felício Rocho, Belo Horizonte, MG, Brazil.
}

\section{Abstract}

Objective: This retrospective study compared the clinical and functional results of single- and double-row surgical techniques for insertional Achilles tendinopathy in the postoperative period.

Methods: In this case series, 29 patients who underwent surgery with one of the two techniques were followed up for one year postoperatively. Data were collected from medical records, imaging exams, and visual analog scale (VAS), Victorian Institute of Sports Assessment-Achilles (VISA-A) questionnaire, 12-item Short Form Health Survey (SF-12), and Foot and Ankle Ability Measure (FAAM) scores.

Results: The following mean values (5\% significance level) were found for single- and double-row techniques, respectively: postoperative VAS (2.9/2.2), FAAM-ADL (71.9/74.4), FAAM-Sports (28.3/29.8), SF-12 physical component (45.2/47.0), SF-12 mental component (44.9/48.2), and VISA-A (72.1/75.9). The complication rate did not differ significantly between the techniques.

Conclusion: No significant differences were found in any of the scores between the two surgical techniques.

Level of Evidence III; Therapeutic Studies, Comparative Retrospective Study.

Keywords: Achilles tendon/surgery; Tendinopathy/surgery; Suture anchors; Suture technique; Orthopedic procedures/methods; Treatment outcome.

\section{Introduction}

Insertional Achilles tendinopathy is attributed to overload, muscle imbalance, poor alignment, decreased blood supply, and tensile strength, and is currently considered multifactorial, involving mechanical, vascular, neural, and genetic factors. The presence of certain systemic diseases and the use of quinolones, statins, corticosteroids, anabolic steroids, and non-hormonal anti-inflammatory drugs also have an influen$c^{(1,2)}$. However, only alcohol abuse and ciprofloxacin have been identified as systemic risk factors for tendinopathy ${ }^{(3,4)}$. The population incidence of insertional Achilles tendinopathy is $3.7 \%$ ( 5 to $18 \%$ among runners), corresponding to $25 \%$ of all
Achilles pathologies. It mainly affects young adults involved in high-demand activities and middle-aged adults with impaired healing potential, impacting performance and quality of life $\mathrm{e}^{(1)}$. It is characterized by pain and edema along the tendon insertion that worsen during exercise.

Studies show that the initial treatment for insertional tendinopathy should be conservative ${ }^{(5-15)}$. Surgery should be considered only when conservative treatment (a maximum of six months) has failed or in cases of persistent pain and limitations in daily and sports activities ${ }^{(1,14,16)}$. Two of the surgical techniques cited in the literature for tendinopathy are single-row repair with two anchors ${ }^{(17)}$ and double-row repair with four anchors ${ }^{(18)}$.

Study performed at the Hospital Felicio Rocho; Hospital Madre Teresa and Hospital Mater Dei, Belo Horizonte, Brazil.

Correspondence: Ricardo Gomes Maciel. 255 Jequitiba St., Ipatinga, MG, Brazil, Zip Code: 35160-306. E-mail: ricardogomesmaciel@gmail.com. Conflicts of interest: Daniel Soares Baumfeld is a consultant and speaker for Arthrex (USA); Tiago Soares Baumfeld is a consultant and speaker for Medartis. The other authors disclaimed that there are no conflicts of interest. Source of funding: none. Date received: March 15, 2021. Date accepted: April 08, 2021. Online: April 30, 2021
How to cite this article: Maciel RG, Castilho RS, Baumfeld DS, Baumfeld TS. A comparative study of single- vs. double-row technique in surgical treatment of insertional Achilles tendinopathy J Foot Ankle. 2021;15(1):8-13. 
The double-row technique was developed to produce greater stability of the tendon insertion and allow earlier rehabilitation without increasing the complication rate ${ }^{(18,19)}$. However, the single-row method also has good functional results and a low rate of complications. McGarvey et al. ${ }^{(20)}$ described a central access technique, finding satisfactory results for functional return and patient satisfaction. Nunley et al. ${ }^{(21)}$ reported a high rate of satisfaction with a method that included central access and repair with two anchors. Therefore, the comparative benefits of single-row vs. double-row techniques are still debated in the literature.

The purpose of this study was to compare patients diagnosed with insertional Achilles tendinopathy who underwent single-row or double-row surgery with respect to pain and function. We hypothesized that, due to its more stable fixation, the double-row technique would provide a safer return to daily and sports activities without increasing the complication rate.

\section{Methods}

This study was approved by the Institutional Review Board and registered on the Plataforma Brasil database under CAAE (Ethics Evaluation Submission Certificate) number: 33931420.8.0000.5128.

This retrospective comparative study assessed the results of single-row or double-row surgery in patients with insertional Achilles tendinopathy who underwent the procedure between July 2014 and January 2020.

The patients were diagnosed by orthopedic foot and ankle specialists and were subject to the following inclusion criteria: agreeing to participate, having been treated with one of the two aforementioned surgical techniques with a minimum follow-up of one year, and having adhered to postoperative guidelines. Patients who did not meet these criteria were excluded from the study. The following variables were evaluated: imaging examinations (radiography of the true anteroposterior projection of the ankle, profile and axial projections of the pre- and postoperative calcaneus, and magnetic resonance imaging of the preoperative ankle), type of postoperative immobilization, and postoperative complications (necrosis, infection, wound dehiscence, tendon rupture, paresthesia, and residual pain). The patients were also questioned about their physical activity (amateur, professional, or sedentary) before and after the procedure. The Visual Analog Scale (VAS) was applied pre- and postoperatively, while the Victorian Institute of Sport Assessment-Achilles (VISA-A) ${ }^{(22)}$, Short Form Health Survey (SF-12), and the Foot and Ankle Ability Measure (FAAM) ${ }^{(23)}$ were applied postoperatively.

Patient data were compared using quantitative statistical analysis to determine the main postoperative differences between the techniques. Statistical tests were performed using $\mathrm{R}$ version 4.0 .3 with a significance level of $5 \%$. The Shapiro-Wilk normality test was used for all scores, with the Wilcoxon test used when normality was rejected.

\section{Surgical techniques}

The two techniques are similar in preparation, positioning, and superficial and deep surgical access, differing only in the use of two or four anchors and their insertion sites. The patient underwent spinal anesthesia and sedation. A tourniquet was placed over the affected ipsilateral thigh and the patient was placed in the prone position. Antisepsis was performed, and the surgical fields were placed in the lower limb. The tourniquet was then inflated. In line with the Achilles tendon, a midline longitudinal incision was made from the calcaneus insertion to approximately $10 \mathrm{~cm}$ proximally. The incision was continued through the subcutaneous tissue to the paratendon, which was opened to reach the Achilles tendon. The tendon was then split with a midline incision, partially released distally in an inverted ' $T$ ' figure, reflected medially and laterally, exposing the entire posterior tuberosity of the calcaneus and the Haglund deformity when present. The degenerated tissue of the Achilles tendon was then debrided. Using an oscillating saw, the calcaneal prominence was resected and the residual edges were smoothed with a file. After resecting the calcaneal prominence and debriding the degenerated Achilles tendon, it was reinserted ${ }^{(24)}$.

In the single-row technique, two $4.5 \mathrm{~mm}$ suture anchors were positioned near the native insertion of the Achilles tendon in the medial and lateral calcaneal tuberosity. Using a free needle, each suture was threaded through the proximal-lateral and proximal-medial portions of the tendon. The anchors were tensioned with the foot in plantar flexion and each suture was tied, reinserting the Achilles tendon. After testing for stability, each suture was threaded through the distal portion of the tendon. The remaining sutures were tied, bringing the distal portion of the tendon closer to the calcaneus. The longitudinal split was sutured with O-Vicryl ${ }^{T M}$. Radiographs were taken to assess the position of the anchor and bone resection. The subcutaneous tissue and skin were closed with a single 2-O Vicryl suture and a single 4-O nylon monofilament suture, respectively. A sterile dressing was applied to the wound, and the leg was immobilized with a dorsal plaster splint or cast placed at 15-20o with the ankle in in the equinus position. The tourniquet was then deflated and the patient was placed in the supine position ${ }^{(13,17)}$. Non-functional immobilization was used during the postoperative period. Weightbearing was prohibited for 4 weeks. The dorsal plaster splint was worn in plantar flexion for the first 2 weeks, after which the sutures were removed. An immobilizing boot with 3 heel wedges was worn from the second to the fourth week. Movements in flexion and extension were then begun to increase the ankle's range of motion, followed by active exercises. From the fourth to sixth week, weightbearing was gradually resumed while wearing the immobilizing boot with wedges. From the sixth to the eighth week, the wedges were gradually removed and physical therapy was begun. Finally, the boot was removed after the eighth week ${ }^{(17)}$.

For the double-row technique, two 4.75-mm BioComposite SwiveLock anchors (Arthrex, Inc., Naples, FL, USA) were inserted into the calcaneus. Two holes were drilled about $1 \mathrm{~cm}$ proximal to the insertion of the Achilles tendon on the calcaneus and central to each half of the tendon. The two anchors, 
loaded with FiberTape (Arthrex) sutures (1 blue and 1 white/ black), were inserted into the proximal holes. Both sutures were threaded through the proximal Achilles tendon on the medial and lateral sides. Two more holes, $2 \mathrm{~cm}$ distal to the proximal anchors, were drilled and threaded using the same technique. The split Achilles tendon was closed with resorbable sutures. The distal anchors were preloaded with a suture tip from the ipsilateral proximal anchor and a suture tail from the contralateral proximal anchor. With the foot in plantar flexion, the suture's tension was adjusted in the distal anchor eyelet. Both distal anchors were tensioned. This process was repeated for the other distal anchor using the remaining 2 suture tails from the proximal row $(12,25,26)$. A single O-Vicryl suture with was used to approximate the debrided area of the Achilles tendon. The subcutaneous tissue and the skin were closed with simple 2-O Vicryl and 4-0 nylon sutures, respectively. Sterile dressing was applied to the wound, and a dorsal plaster splint or cast was placed at $15-20^{\circ}$ with the ankle in in the equinus position. The tourniquet was then deflated and the patient was placed in the supine position. Functional immobilization was used during the postoperative period. The patients wore an immobilizing boot with 3 heel wedges, and weightbearing was allowed after 1 week. The wedges were gradually removed until the sixth week, when the boot was removed. The suture was removed after 2 weeks. Physical therapy was begun after approximately 3 to 4 weeks ${ }^{(24)}$.

\section{Results}

This study included 29 patients with insertional Achilles tendinopathy, of whom 14 (48\%) underwent surgery with the single-row technique and 15 (52\%) with the double-row technique between July 2014 and January 2020. The sample included 15 (52\%) women, the mean age was 54 years (3379), and there was a minimum follow-up of one year after the procedure.

Regarding sports activities, 14 (48\%) patients reported none, 14 reported an amateur level, and 1 reported a professional level. Only 5 patients had complications: there were 2 (7\%) cases of residual pain ( 1 in each group), 1 (3\%) case each of wound dehiscence and paresthesia in the double-row group, and 1 (3\%) case of superficial infection in the single-row group. The remaining 24 (83\%) patients had no complications.

The pre- and postoperative VAS values, as well as the difference observed between the two time points were graphed (Figure 1). The following values were obtained for the singleand double-row techniques, respectively: preoperative VAS (8.7/7.8) and postoperative VAS (2.9/2.2). There were no significant differences in VAS score between the groups.

The VISA-A (Figure 2), FAAM (Figure 3), the SF-12 physical (Figure 4) and SF-12 mental (Figure 5) components were distributed as follows for the single-row and double-row techniques, respectively: VISA-A (72.1/75.9); FAAM ADL (71.9/74.4); FAAM sports (28.3/29.8); the SF-12 physical (45.2/47.0) and SF-12 mental (44.9/48.2). Thus, there were no significant differences (at a significance level of $5 \%$ ) in these scores between the procedures.

\section{Discussion}

This study compared the functional results of single-row and double-row surgical techniques for treating insertional tendinopathy of the Achilles tendon. No statistically significant differences were found between the techniques from a clinical and functional point of view.

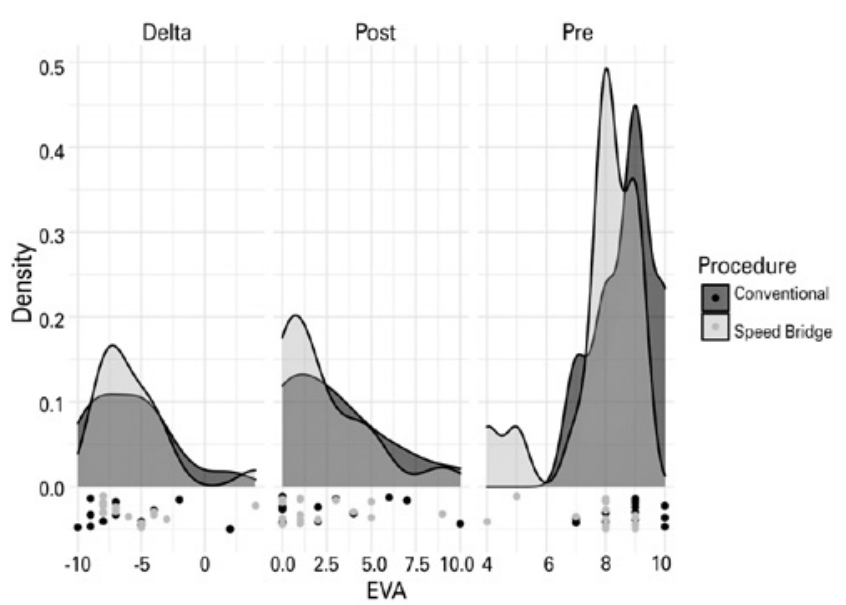

Figure 1. Visual Analog Scale results. The curves and darker points represent data collected from the single-row group, while the lighter tones represent the double-row group. The curves represent the density of the values according to row, while the points are the exact values collected.

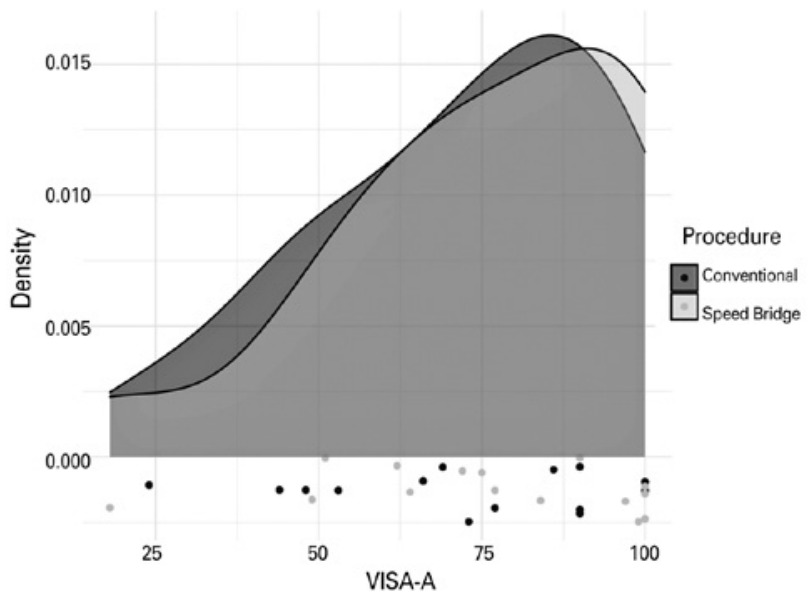

Figure 2. VISA-A results. The curves and darker points represent data collected from the single-row group, while the lighter tones represent the double-row group. The curves represent the density of the values according to row, while the points are the exact values collected. 


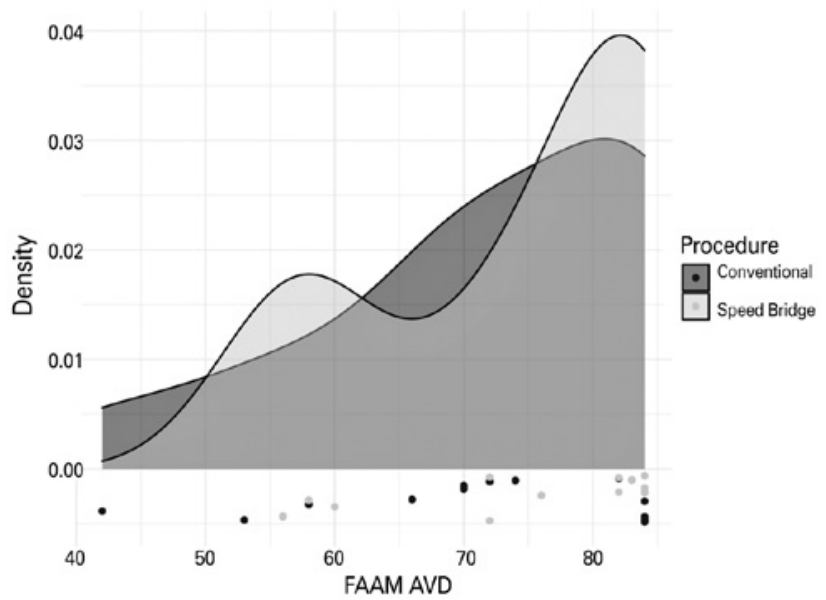

Figure 3. FAAM results. The curves and darker points represent data collected from the single-row group, while the lighter tones represent the double-row group. The curves represent the density of the values according to row, while the points are the exact values collected.

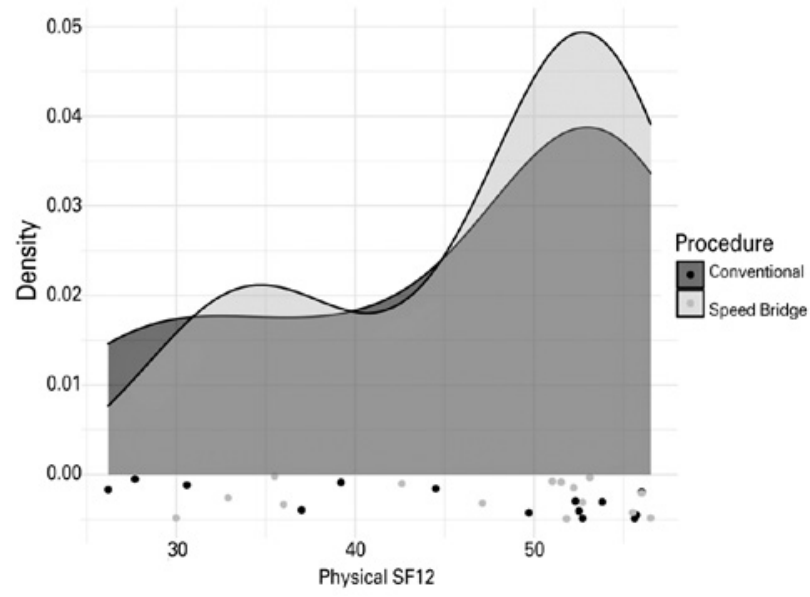

Figure 4. SF-12 physical component results. The curves and darker points represent data collected from the single-row group, while the lighter tones represent the double-row group. The curves represent the density of the values according to row, while the points are the exact values collected.

Regarding the VAS, Rigby et al. ${ }^{(24)}$ carried out a study with 43 patients who underwent the double-row technique, finding mean values of 6.8 and 1.3 in the pre- and postoperative period, respectively. Using the single-row technique in 36 patients, Xia et al. ${ }^{(13)}$ found mean pre- and postoperative VAS values of 7.8 and 1.8 , respectively. In the current study, the mean pre- and postoperative VAS values for the single-row technique were 8.7 and 2.9, respectively, and 7.8 and 2.2 for the double-row technique, respectively. As in the above-mentioned studies, the techniques led to improved, but not significantly different, pain results in the postoperative period.

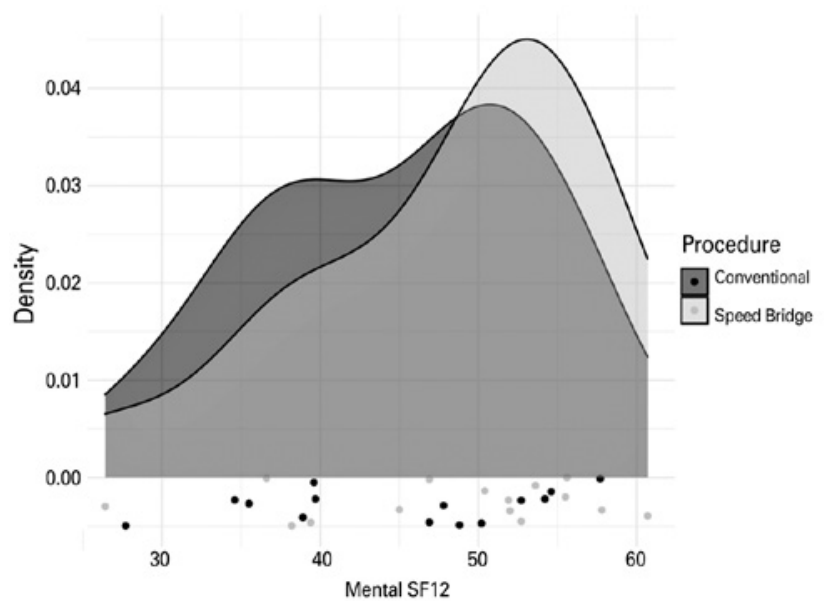

Figure 5. SF-12 mental component results. The curves and darker points represent data collected from the single-row group, while the lighter tones represent the double-row group. The curves represent the density of the values according to row, while the points are the exact values collected.

Table 1. Descriptive statistics of numerical variables according to surgical procedure

\begin{tabular}{lcccccc} 
Variable & Procedure & Min. & Mean & Median & Max. & SD \\
\hline VAS & Single-row & 0 & 2.9 & 2 & 10 & 3.08 \\
postoperative & Double-row & 0 & 2.2 & 1 & 9 & 2.57 \\
\cline { 2 - 7 } VAS & Single-row & 7 & 8.7 & 9 & 10 & 0.99 \\
preoperative & Double-row & 4 & 7.8 & 8 & 9 & 1.47 \\
& Single-row & 42 & 71.9 & 73 & 84 & 13.37 \\
FAAM ADL & Double-row & 56 & 74.4 & 82 & 84 & 11.34 \\
\multirow{2}{*}{ FAAM sports } & Single-row & 22 & 28.3 & 31 & 32 & 5.51 \\
& Double-row & 23 & 29.8 & 32 & 32 & 3.90 \\
SF-12 physical & Single-row & 26 & 45.2 & 51 & 56 & 11.00 \\
& Double-row & 30 & 47.0 & 52 & 56 & 9.10 \\
\hline \multirow{2}{*}{ SF-12 mental } & Single-row & 28 & 44.9 & 47 & 58 & 8.95 \\
& Double-row & 26 & 48.2 & 52 & 61 & 9.40 \\
\multirow{2}{*}{ VISA-A } & Single-row & 24 & 72.1 & 75 & 100 & 22.92 \\
& Double-row & 18 & 75.9 & 77 & 100 & 23.95 \\
\hline
\end{tabular}

ADL: activities of daily living; FAAM: Foot and Ankle Ability Measure; SD: Standard deviation; SF-12: 12-item Short Form Health Survey; VAS: Visual Analog Scale; VISA-A: Victorian Institute of Sports Assessment-Achilles questionnaire.

The VISA-A score, which is used to assess Achilles tendon disorders and their functional impact, is easy to apply clinically and provides a reliable index of the severity of tendinopathy ${ }^{(16,27,28)}$. Due to its brevity and performance, the SF-12 score, a general assessment of quality of life related to physical and mental health, is widely used in clinical trials and to assess routine results $^{(29,30)}$. The FAAM score is a reliable and valid measure of physical function in individuals with musculoskeletal disorders of the foot and ankle ${ }^{(31,32)}$. There is a lack of studies in the scientific literature associating these scores and surgical techniques. In this study, no significant differences were found between the techniques in the postoperative period. 
Biomechanical studies have found conflicting results about the best surgical technique. For example, Beitzel et al. ${ }^{(33)}$ concluded that the double-row technique has a greater contact area and tolerates greater tension in stress testing than the single-row technique, which corroborates the results of a previous study by an orthopedic implant company ${ }^{(34)}$. However, a recent cadaver study ${ }^{(35)}$, as well as Pilson et al. ${ }^{(36)}$, found that the double-row technique could not tolerate a greater load before failure.

We found different types of postoperative immobilization protocols used in the literature. In Gillis and Lin ${ }^{(26)}$, the patients went 4 weeks without weightbearing, progressively returned to weightbearing with immobilizing boots and wedges, followed by another 4 weeks of immobilizing boots without wedges. In Rigby et al. ${ }^{(24)}$, the patients returned to weightbearing based on age, weight, comorbidities, activity level and concomitant procedures, varying from immediate weightbearing with an immobilizing boot to no weightbearing for several weeks. In Zhuang et al. ${ }^{(12)}$ the patients went 4 weeks without weightbearing, followed by partial weightbearing with an immobilizing boot and wedges that were removed gradually over 6 weeks, after which full weightbearing was allowed. Thus, non-functional immobilization was used even with the double-row technique. Whereas, in Xia et al.(13), a study of the single-row technique, the patients went 2 weeks without weightbearing, followed by 4 weeks of partial weightbearing with an immobilizing boot, followed by boot removal and full weightbearing at 6 weeks. Despite their different immobilization protocols, all of these studies had good postoperative results. In the present study, postoperative immobilization was pre- dominantly functional in the double-row group since this technique was expected to be more resistant. Therefore, these patients were prescribed earlier mobilization and weight bearing despite the conflicting results of the above-mentioned biomechanical studies.

Highlander and Greenhagen(37) conducted a systematic review on wound complications in posterior leg incisions, finding no difference in the incidence of complications between midline and medial incisions, with the medial incision allowing better visualization for tendon resection and reinsertion. Several studies have shown that central posterior access resulted in a low rate of complications for these techniques ${ }^{(12,20,25,38)}$. The present study also had a low rate of complications for both techniques: importantly, no cases of postoperative tendon rupture occurred.

This study's weaknesses include the small number of patients, its retrospective and non-randomized design, the fact that the postoperative immobilization type differed between techniques, the fact that all scores were not also collected in the preoperative period, and the inclusion of professional athletes, who may place greater stress on the tendon. Regarding its strengths, it was a comparative study, it included a minimum follow-up of one year, and it used validated scales, both general and specific.

According to the assessed scales and postoperative complication rates, neither technique was superior. Therefore, new studies with larger samples, participants with high functional demand, and use of similar postoperative immobilization for both techniques are needed to evaluate our hypothesis.

Authors' contributions: Each author contributed individually and significantly to the development of this article: RGM *(https://orcid.org/O000-00025366-5394) participated in the review process, data collection; statistical analysis, bibliographic review, survey of medical records, article formatting; RSC *(https://orcid.org/0000-0002-8930-7046) participated in the review process, performed the surgeries; DSB *(https://orcid.org/0000-0001-5404-2132) conceived and planned the activities that led to the study, participated in the review process, performed the surgeries; TSB *(https://orcid.org/O000-00019244-5194) participated in the review process, performed the surgeries. All authors read and approved the final manuscript. ${ }^{*}$ ORCID (Open Researcher and Contributor ID) iD.

\section{References}

1. Mansur NSB, Fonseca LF, Matsunaga FT, Baumfeld DS, Nery CAS, Tamaoki MJS. Achilles tendon lesions - Part 1: tendinopathies. Rev Bras Ortop (Sao Paulo). 2020;55(6):657-64.

2. Waldecker $U$, Hofmann G, Drewitz S. Epidemiologic investigation of 1394 feet: coincidence of hindfoot malalignment and Achilles tendon disorders. Foot Ankle Surg. 2012;18(2):119-23.

3. van der Vlist AC, Breda SJ, Oei EHG, Verhaar JAN, de Vos RJ. Clinical risk factors for Achilles tendinopathy: a systematic review. Br J Sports Med. 2019;53(21):1352-61.

4. Marie I, Delafenêtre H, Massy N, Thuillez C, Noblet C; Network of the French Pharmacovigilance Centers. Tendinous disorders attributed to statins: a study on ninety-six spontaneous reports in the period 1990-2005 and review of the literature. Arthritis Rheum. 2008;59(3):367-72.
5. Stania M, Juras G, Chmielewska D, Polak A, Kucio C, Król P. Extracorporeal shock wave therapy for Achilles tendinopathy. Biomed Res Int. 2019;2019:3086910.

6. Fan Y, Feng Z, Cao J, Fu W. Efficacy of extracorporeal shock wave therapy for Achilles tendinopathy: a meta-analysis. Orthop J Sports Med. 2020;8(2):2325967120903430.

7. Alfredson H, Pietilä T, Jonsson P, Lorentzon R. Heavy-load eccentric calf muscle training for the treatment of chronic Achilles tendinosis. Am J Sports Med. 1998;26(3):360-6.

8. Chimenti RL, Cychosz CC, Hall MM, Phisitkul P. Current Concepts Review Update: Insertional Achilles Tendinopathy. Foot Ankle Int. 2017;38(10):1160-9.

9. Mansur NS, Faloppa F, Belloti JC, Ingham SJ, Matsunaga FT, Santos PR, et al. Shock wave therapy associated with eccentric 
strengthening versus isolated eccentric strengthening for Achilles insertional tendinopathy treatment: a double-blinded randomised clinical trial protocol. BMJ Open. 2017;7(1):e013332.

10. Arnal-Gómez A, Espí-López GV, Cano-Heras D, Muñoz-Gómez E, Balbastre Tejedor I, Ramírez Iñiguez-de la Torrez MV, et al. Efficacy of eccentric exercise as a treatment for Achilles Tendinopathy: literature review. Arch Prev Riesgos Labor. 2020;23(2):211-33.

11. Caudell GM. Insertional Achilles tendinopathy. Clin Podiatr Med Surg. 2017;34(2):195-205.

12. Zhuang Z, Yang Y, Chhantyal K, Chen J, Yuan G, Ni Y, et al. Central tendon-splitting approach and double row suturing for the treatment of insertional Achilles tendinopathy. Biomed Res Int. 2019;2019:4920647.

13. Xia Z, Yew AKS, Zhang TK, Su HCD, Ng YCS, Rikhraj IS. Surgical correction of haglund's triad using a central tendon-splitting approach: a retrospective outcomes study. J Foot Ankle Surg. 2017;56(6):1132-38.

14. Barg A, Ludwig T. Surgical strategies for the treatment of insertional achilles tendinopathy. Foot Ankle Clin. 2019;24(3):533-59.

15. Aronow MS. Posterior heel pain (retrocalcaneal bursitis, insertional and noninsertional Achilles tendinopathy). Clin Podiatr Med Surg. 2005;22(1):19-43.

16. Maffulli N, Longo UG, Kadakia A, Spiezia F. Achilles tendinopathy. Foot Ankle Surg. 2020;26(3):240-9.

17. Ricci AG, Stewart M, Thompson D, Watson BC, Ashmyan R. The central-splitting approach for Achilles insertional tendinopathy and Haglund deformity. JBJS Essent Surg Tech. 2020;10(1):e0035.

18. Witt BL, Hyer CF. Achilles tendon reattachment after surgical treatment of insertional tendinosis using the suture bridge technique: a case series. J Foot Ankle Surg. 2012;51(4):487-93.

19. Mineta K, Suzue N, Matsuura T, Sairyo K. Efficacy of Achilles suture bridge technique for insertional Achilles tendinosis in an obese and athletic patient. J Med Invest. 2016;63(3-4):310-4.

20. McGarvey WC, Palumbo RC, Baxter DE, Leibman BD. Insertional Achilles tendinosis: surgical treatment through a central tendon splitting approach. Foot Ankle Int. 2002;23(1):19-25.

21. Nunley JA, Ruskin G, Horst F. Long-term clinical outcomes following the central incision technique for insertional Achilles tendinopathy. Foot Ankle Int. 2011;32(9):850-5.

22. de Mesquita GN, de Oliveira MNM, Matoso AER, de Moura Filho $A G$, de Oliveira RR. Cross-cultural adaptation and measurement properties of the Brazilian Portuguese version of the Victorian Institute of Sport Assessment-Achilles (VISA-A) Questionnaire. J Orthop Sports Phys Ther. 2018;48(7):567-73

23. Moreira TS, Magalhães Lde C, Silva RD, Martin RL, Resende MA. Translation, cross-cultural adaptation and validity of the Brazilian version of the Foot and Ankle Ability Measure questionnaire. Disabil Rehabil. 2016;38(25):2479-90.

24. Rigby RB, Cottom JM, Vora A. Early weightbearing using Achilles suture bridge technique for insertional Achilles tendinosis: a review of 43 patients. J Foot Ankle Surg. 2013;52(5):575-9.
25. Greenhagen RM, Shinabarger AB, Pearson KT, Burns PR. Intermediate and long-term outcomes of the suture bridge technique for the management of insertional Achilles tendinopathy Foot Ankle Spec. 2013;6(3):185-90.

26. Gillis CT, Lin JS. Use of a central splitting approach and near complete detachment for insertional calcific Achilles tendinopathy repaired with an Achilles bridging suture. J Foot Ankle Surg. 2016; 55(2):235-9.

27. Palazón-Bru A, Tomás-Rodríguez MI, Mares-García E, Gil-Guillén VF. A reliability generalization meta-analysis of the Victorian Institute of Sport Assessment Scalel for Achilles tendinopathy (VISA-A). Foot Ankle Int. 2019;40(4):430-8.

28. Robinson JM, Cook JL, Purdam C, Visentini PJ, Ross J, Maffulli $\mathrm{N}$, et al. The VISA-A questionnaire: a valid and reliable index of the clinical severity of Achilles tendinopathy. Br J Sports Med. 2001;35(5):335-41.

29. Brazier JE, Roberts J. The estimation of a preference-based measure of health from the SF-12. Med Care. 2004;42(9):851-9.

30. Ceravolo ML, Gaida JE, Keegan RJ. Quality-of-life in Achilles tendinopathy: an exploratory study. Clin J Sport Med. 2020; 30(5):495-502

31. Martin RL, Irrgang JJ, Burdett RG, Conti SF, Van Swearingen JM. Evidence of validity for the Foot and Ankle Ability Measure (FAAM). Foot Ankle Int. 2005;26(11):968-83.

32. Mazaheri M, Salavati M, Negahban H, Sohani SM, Taghizadeh F, Feizi A, et al. Reliability and validity of the Persian version of Foot and Ankle Ability Measure (FAAM) to measure functional limitations in patients with foot and ankle disorders. Osteoarthritis Cartilage. 2010;18(6):755-9.

33. Beitzel K, Mazzocca AD, Obopilwe E, Boyle JW, McWilliam J, Rincon L, et al. Biomechanical properties of double- and single-row suture anchor repair for surgical treatment of insertional Achilles tendinopathy. Am J Sports Med. 2013;41(7):1642-8.

34. Amendola N, Boyle J, Mazzocca A, Obopilwe E, Rincon L, McWilliam J, et al. Biomechanical testing of distal Achilles fixation using suture anchors: single row vs. SutureBridge (Internet). Naples, Florida: Arthrex Inc.; 2007. Available at: https://www. arthrex.com/foot-ankle/suturebridge

35. Lakey E, Kumparatana P, Moon DK, Morales J, Anderson SE, Baldin $\mathrm{T}$, et al. Biomechanical comparison of all-soft suture anchor singlerow vs double-row bridging construct for insertional Achilles tendinopathy. Foot Ankle Int. 2021;42(2):215-23.

36. Pilson H, Brown P, Stitzel J, Scott A. Single-row versus double-row repair of the distal Achilles tendon: a biomechanical comparison. J Foot Ankle Surg. 2012;51(6):762-6.

37. Highlander P, Greenhagen RM. Wound complications with posterior midline and posterior medial leg incisions: a systematic review. Foot Ankle Spec. 2011;4(6):361-9.

38. Ahn JH, Ahn CY, Byun CH, Kim YC. Operative treatment of Haglund syndrome with central Achilles tendon-splitting approach. J Foot Ankle Surg. 2015;54(6):1053-6. 